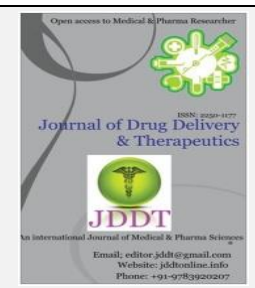

\title{
Generalizability Probability: Importance in Clinical Research
}

\section{R. Santosh Kumar and S. Sai Kiran}

GITAM Institute of Pharmacy, GITAM (Deemed To Be University) Rushikonda, Visakhapatnam-530045, Andhra Pradesh, India

\begin{abstract}
International conference on harmonization (ICH) suggested that bridging study is important to extrapolate clinical results. and also us food and drug administration requires Substantial evidences regarding safety. The review explains about different approaches that are necessary for obtaining clinical results, various clinical results were performed between similar patient population within same or differe nt regions. moreover no criteria on the evaluation of similarity of clinical results between regions is given .It is important in regulatory submission of new drug application. Mainly this review explains about how clinical results are reproducible.
\end{abstract}

Keywords: ICH, Generalizability, probability Reproducibility, clinical trails, bridging study.

Article Info: Received 16 July 2019; $\quad$ Review Completed 13 Aug 2019; $\quad$ Accepted 24 Aug 2019; Available online 15 Oct 2019

\section{Cite this article as:}

Santosh Kumar R, Sai Kiran S, Generalizability Probability: Importance in Clinical Research, Journal of Drug Delivery and Therapeutics. 2019; 9(5-s):162-163 http://dx.doi.org/10.22270/jddt.v9i5-s.3587

\section{*Address for Correspondence:}

R. Santosh Kumar, GITAM Institute of Pharmacy, GITAM (Deemed to be University), Gandhinagar, Visakhapatnam530045, Andhra Pradesh, INDIA.

\section{INTRODUCTION:}

US food and drug administration (USFDA) requires well controlled clinical trials for providing substantial evidences and also regarding the safety under investigation. The main purpose of requiring these two clinical trials is to assure the reproducibility and also to provide information regard in generalizability. It can be defined as extension of research finding and conclusions.

The clinical trials can be performed to other similar patient population with in same region or from region to region, which means drugs to be approved by same region or from different regions etc.

ICH international conference on Harmonisation recommends bridging studies to generate limited amount of clinical data in new region mainly to extrapolate clinical data between two regions. For assurance of generalizability, it is very essential and necessary to determine whether a clinical data providing positive results along with substantial evidence.

This review also introduces concepts of generalizability of positive clinical trials is also introduced. In these review several methods were discussed for evaluation of generalizability which includes Frequentist approach and Bayesian approach[1] .

\section{GENERALIZABILITY:}

Clinical trails were to be performed for considering the situation, but due to differences in ethnic factors second clinical and first clinical results were slightly different.

Mainly it includes the differences in mean and variance. Sometimes both mean variance of primary endpoints too. Suppose in first clinical trial if we take old parents and in second clinical trail if we choose adults then the two results will be different.

Suppose both 1 and 2 clinical trails of same population then reproducibility will be different. So for this the scientist named SHAO and CHOW defined about reproducibility and pprobability[3].To access the same clinical results we have to use the first clinical data to second one so that the results may be same and common.

\section{FREQUENTIST APPROACH:[2]}

In two parallel group design, may $\mu_{1} \mu_{2}$ be population mean difference and $\sigma 2$ be common population variance of first trail

In case if the second trail population, mean difference can change to $\mu_{1}-\mu_{2}+\mathrm{e}$ and population variance changes to $c^{2} \sigma 2$.

In that $c>0\left[\mu_{1}-\mu_{2}\right] \backslash \sigma$ is signal to noise ratio.

Then the signal to noise ratio for population difference in second trial is 


\section{$\left\lfloor\mu_{1}-\underline{\mu}_{2}+\mathrm{e} \mid=\perp \Delta(\mu-\mu)\right.$}

$\mathrm{C} \sigma$

$\sigma$

Where

$$
\Delta=\underline{1+e \backslash\left(\mu_{1}-\underline{\mu_{2}}\right.}
$$

C

It is a measure of change in signal to noise ratio for population different patients are randomly assigned for treatment group then the power for second trail is :

$\left.1-\mathrm{T}_{\mathrm{n}-2}\left(\mathrm{t}_{0.975 ; \mathrm{n}-2} \mid \Delta \theta\right)+\mathrm{T}_{\mathrm{n}-2}\right)(\mathrm{t} 0.975 ; \mathrm{n}-2 \mid \Delta \theta)$

$\mathrm{T}_{\mathrm{n}-2}(\Delta \theta)$ is non central $\mathrm{t}$-distribution with no degrees of freedom and non centrality parameter $\Delta \theta$.

$$
\theta=\mu 1-\mu 2 / \sigma \sqrt{1 / \mathrm{n} 1+1 / \mathrm{n} 2}
$$

In these $\mathrm{x}_{1} \mathrm{~s}$ and $\mathrm{s}^{2} 2 . \mathrm{s}$ are sample means variance of first trial data base reproducibility, probability in clinical research can be obtained with $\mathrm{T}$ and replaced by $\Delta \mathrm{t}$.

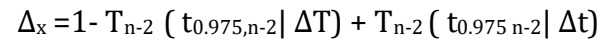

Ggeneralizability, probability, based on approach as outlined in entry

Rreproducibility, probability in clinical research is given by

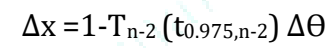

Values of $\mathrm{p} \Delta$ can be obtained using 2 and 3 tablets in entry of reproducibility and probability in clinical research.

BAYESIAN APPROACH: [3]

Based on approach given by

$$
\Pi\left(\delta / \gamma^{2}, x\right) \pi\left(\gamma^{2} / x\right)
$$

$\phi=$ density function of normal distribution
$T$ is given by

$$
\pi(\gamma 2 \mid x)=f(u)
$$

We may consider avg $p \Delta=\int p \Delta \pi(\Delta) d \Delta$.

\section{APPLICATIONS:}

Information about bridging studies can be found in ICH GUIDELINES in detail. It is useful in regulatory submission for supplement new drug application mmainly in clinical development after the investigation drug product has been shown to be effective and safe with respect to patient populationn.It is often of interest to study how likely the clinical result is reproducible in similar but slightly different in patient population.

\section{CONCLUSION:}

It is important to determine whether clinical results can be generalized. From patient population to another slight different population with in same region. These approaches are used for helpful of qualitative analysis of generalizability probabilities. Also it is important to determine clinical trail that is produced by substantial evidences are also helpful in assurance of generalizability. Difference varies in mean variance and also both mean and variance of primary endpoints, which are used to obtain generalizability in case study. Designs with slight common variance on the other cases are illustrated. These are generally used for approval of new drug product.

\section{REFERENCES:}

1 FDA. Guideline for format and content of the clinical selections of the Drug Research. The united states food and drug approval Rockville,MD,USA,1988

$2 \mathrm{ICH}$. Ethnic factors in the of foreign tripartite international conference On harmonization 1998,E5.

3 Shao, J; Chow, S.C, Reproducibility probability in Stat. Med. 2002, 21,1727-1742. 